Research Article

\title{
The Abnormal Phenomena of Entropy Weighting Method in the Dynamic Evaluation of Agricultural Water Conservation
}

\author{
Liangzhen Zhu $\mathbb{( D},{ }^{1}$ Xigang Xing $\left(\mathbb{D},{ }^{2}\right.$ and Feng Yan $\mathbb{D}^{3}$ \\ ${ }^{1}$ School Hydrology and Water Resources, Hohai University, Nanjing 210098, China \\ ${ }^{2}$ General Institute of Water Resources and Hydropower Planning and Design, Ministry of Water Resources, Beijing 100120, China \\ ${ }^{3}$ School of Civil Engineering and Architecture, Nanchang University, Nanchang 330031, China
}

Correspondence should be addressed to Feng Yan; yfmilan@163.com

Received 10 April 2021; Revised 28 July 2021; Accepted 30 July 2021; Published 13 August 2021

Academic Editor: António M. Lopes

Copyright ( $) 2021$ Liangzhen Zhu et al. This is an open access article distributed under the Creative Commons Attribution License, which permits unrestricted use, distribution, and reproduction in any medium, provided the original work is properly cited.

\begin{abstract}
Through a simple and intuitive example of the agricultural water conservation assessment in 3 provinces, China, the abnormal phenomena of the entropy weighting method (EWM) in the dynamic evaluation are revealed. The results show the following. (i) The irrigation water efficiency percentages (IWEPs) of these 3 provinces are improved from $53 \%, 53 \%$, and $55 \%$ to $55 \%, 56 \%$, and $56 \%$, respectively. And their water-saving irrigation projects percentages (WSIPPs) are improved from $40 \%, 41 \%$, and $41 \%$ to $42 \%$, $42 \%$, and $42 \%$, respectively. However, their comprehensive agricultural conservation indices deteriorate from $52.11,52.45$, and 56.1 to $46.07,46.74$, and 48.57, respectively. (ii) EWM leads to the following paradox in the dynamic evaluation. All the indicators show improving trends, but the comprehensive evaluation results show a deteriorating trend. (iii) These abnormal phenomena of EWM are induced by that though all the indicators are improved, the discrimination of the worse indicators becomes larger while the discrimination of the better indicators becomes smaller. (iv) The abnormal phenomena of EWM in dynamic evaluation can be avoided by the trend analysis of the observation data and entropy values. When all the indicators have improvement trends, but the entropies of the better indicators are increasing and the entropies of the worse indicators are decreasing, EWM should not be used for assigning weights.
\end{abstract}

\section{Introduction}

The entropy weight method (EWM) is a widely used weighting method in multi-index evaluation. It assigns weight by calculating the amount of information of indicators. The higher the information amount is, the greater the weight is; and vice versa $[1,2]$. Compared with analytic hierarchy process method, the major advantage of EWM is that it eliminates the interference of subjective factors and ensures the objectivity of weight $[3,4]$.

In the previous literature, the validity of EWM was generally considered to be beyond doubt [5-7]. However, Yan et al. [8], Qian et al. [9], and Zhu et al. [10] have challenged this view in recent years. Yan et al. [8] pointed out that the information amount could not correctly represent the importance of the measured values which concentrated in the worst grade. Qian et al. [9] and Zhu et al.
[10] further found that when the observation data contained too many 0 values, the EWM had distortion in the standardization process. To solve these problems, Yan et al. [8], Qian et al. [9], and Zhu et al. [10] all proposed that the rationality of the EWM should be tested according to the distribution of the measured values.

It is noteworthy that all the studies of Yan et al. [8], Qian et al. [9], and Zhu et al. [10] focus on the distortion phenomenon of EWM in static evaluation, in which the weight parameters and observation data are not changed with time. However, in many multi-index evaluation problems, the status of indicators varies about time, which is known as dynamic evaluation $[11,12]$. Through large amounts of practices, we find that EWM has another abnormal phenomenon in the dynamic evaluation. The reasonable entropy weight results may lead to wrong comprehensive evaluation conclusions. In this study, we will reveal this phenomenon 
with a simple and intuitive dynamic evaluation of agricultural water conservation.

\section{Materials and Methods}

2.1. Dynamic Evaluation of Agricultural Water Conservation. Water is the survival basis for human beings and an indispensable natural resource for social development [13]. However, with the population expansion and economic development, the water resources consumed by human have increased dramatically in recent years, which caused great pressure on the aquatic ecosystems [13]. According to "the Chinese water conservation evaluation report" made by China Renewable Energy Engineering Institute (CREEI), about $70 \%$ of the water consumed by society is used for agricultural irrigation [13]. Therefore, the agricultural water conservation has become a researching priority in the global water resources management.

According to the research of CREEI, less than $30 \%$ of the irrigation water is utilized by crops and the remaining $70 \%$ is consumed by the evaporation and leakage in irrigation projects [13]. Therefore, CREEI uses irrigation water efficiency percentage (IWEP) and water-saving irrigation projects percentage (WSIPP) to assess the status of agricultural water conservation [13]. IWEP is defined as the proportion of the water used by crops to the total irrigation water; and WSIPP is the proportion of water-saving irrigation projects to the total irrigation projects [13]. CREEI classifies the agricultural water conservation condition into 4 levels, which is illustrated in Table 1 [13].

As the government continues to invest in agriculture, the agricultural water conservation condition in China is improving year by year. As a result, the data of each indicator change over time, too. The value of the $j$ th indicator of the $i$ th province in the $k$ th year is denoted as $x_{i j}\left(t_{k}\right)$. To eliminate the differences among the dimensions of evaluation indicators, the water-saving index $y_{i j}\left(t_{k}\right)$ is defined as follows [13].

For IWEP,

$$
y_{i 1}\left(t_{k}\right)= \begin{cases}75+25 \cdot \frac{x_{i 1}\left(t_{k}\right)-60 \%}{40 \%}, & x_{i 1}\left(t_{k}\right) \geq 60 \%, \\ 50+25 \cdot \frac{x_{i 1}\left(t_{k}\right)-50 \%}{10 \%}, & 50 \% \leq x_{i 1}\left(t_{k}\right)<60 \%, \\ 25+25 \cdot \frac{x_{i 1}\left(t_{k}\right)-45 \%}{5 \%}, & 45 \% \leq x_{i 1}\left(t_{k}\right)<50 \%, \\ 25 \cdot \frac{x_{i 1}\left(t_{k}\right)}{45 \%}, & x_{i 1}\left(t_{k}\right)<45 \%,\end{cases}
$$

and for WSIPP,
TABLE 1: Threshold values for indicators in agricultural water conservation evaluation.

\begin{tabular}{lcccc}
\hline Indicators & Excellent & Good & Medium & Backward \\
\hline WWEP & $60 \%-100 \%$ & $50 \%-60 \%$ & $45 \%-50 \%$ & $0 \%-45 \%$ \\
$70 \%-100 \%$ & $50 \%-70 \%$ & $30 \%-50 \%$ & $0 \%-30 \%$ \\
\hline$y_{i 2}\left(t_{k}\right)=\left\{\begin{array}{cl}75+25 \cdot \frac{x_{i 2}\left(t_{k}\right)-70 \%}{30 \%}, & x_{i 2}\left(t_{k}\right) \geq 70 \%, \\
50+25 \cdot \frac{x_{i 2}\left(t_{k}\right)-50 \%}{20 \%}, & 50 \% \leq x_{i 2}\left(t_{k}\right)<70 \%, \\
25+25 \cdot \frac{x_{i 2}\left(t_{k}\right)-30 \%}{20 \%}, & 30 \% \leq x_{i 2}\left(t_{k}\right)<50 \%, \\
25 \cdot \frac{x_{i 2}\left(t_{k}\right)}{30 \%}, & x_{i 2}\left(t_{k}\right)<30 \% .\end{array}\right.$
\end{tabular}

Obviously, $y_{i j}\left(t_{k}\right)$ is the larger the better; and the judgment intervals corresponding to excellent, good, medium, and backward are [75,100], [50,75), [25,50), and [0,25), respectively [13].

The comprehensive agricultural conservation index of the $i$ th region in the $k$ th year can be quantified by $z_{i}\left(t_{k}\right)$, which is calculated by [13] the following:

$$
z_{i}\left(t_{k}\right)=\sum_{j=1}^{2}\left\{w_{j}\left(t_{k}\right) \cdot y_{i j}\left(t_{k}\right)\right\}
$$

where $w_{j}\left(t_{k}\right)$ is the weight of the $j$ th indicator in the $k$ th year [13]. Because the values of indicators change with time, their weights have interannual differences, too.

2.2. EWM in Dynamic Evaluation. EWM assigns weights based on the information amount of data [8-10]. The larger the dispersion is, the more information it contains and a higher weight is assigned [8-10]. On the contrary, the smaller the dispersion is, the less information it contains and a lower weight is assigned [8-10].

The first step in the EWM is standardization. For the income-type indicators [8-10],

$$
p_{i j}\left(t_{k}\right)=\frac{x_{i j}\left(t_{k}\right)-\min _{i=1,2, \ldots, m} x_{i j}\left(t_{k}\right)}{\max _{i=1,2, \ldots, m} x_{i j}\left(t_{k}\right)-\min _{i=1,2, \ldots, m} x_{i j}\left(t_{k}\right)} .
$$

And, for the cost-type indicators [8-10],

$$
p_{i j}\left(t_{k}\right)=\frac{\max _{i=1,2, \ldots, m} x_{i j}\left(t_{k}\right)-x_{i j}\left(t_{k}\right)}{\max _{i=1,2, \ldots, m} x_{i j}\left(t_{k}\right)-\min _{i=1,2, \ldots, m} x_{i j}\left(t_{k}\right)} .
$$

The entropy value of the $j$ th indicator in the $k$ th year is $H_{j}\left(t_{k}\right)[8-10]:$ 


$$
H_{j}\left(t_{k}\right)=\frac{-\sum_{i=1}^{m}\left(f_{i j}\left(t_{k}\right) \cdot \ln f_{i j}\left(t_{k}\right)\right)}{\ln m},
$$

where $f_{i j}\left(t_{k}\right)=p_{i j}\left(t_{k}\right) / \sum_{i=1}^{m} p_{i j}\left(t_{k}\right)$. According to L'Hospital's rule, when $f_{i j}\left(t_{k}\right)=0, f_{i j}\left(t_{k}\right) \cdot \ln f_{i j}\left(t_{k}\right)$ is defined to be 0 [8-10].

The weight parameter $w_{j}\left(t_{k}\right)$ is generated as follows [8-10]:

$$
w_{j}\left(t_{k}\right)=\frac{1-H_{j}\left(t_{k}\right)}{n-\sum_{j=1}^{n} H_{j}\left(t_{k}\right)} .
$$

It is easy to find that in the dynamic multi-index comprehensive evaluation, the EWM assign weights are only based on the measured values of evaluation indicators in different years, which eliminates the interference of subjective factors and ensures the objectivity of weights.

\section{Results and Discussion}

3.1. Statistical Characteristics of Data. In this study, a simple and intuitive example was chosen from a large number of practices and Monte Carlo simulations. It was assumed that in 2019 and 2020, the agricultural conservation condition in three provinces is evaluated; and the data of each indicator are shown in Table 2.

According to Table 1, it is easy to find that all the data of IWEP in 2019 and 2020 belong to the "good" grade and all the values of WSIPP belong to the "medium" grade. On the whole, the grades of the two indicators are similar; and none of them concentrates in the worst grade, which avoids the distortion phenomenon pointed out by Yan et al. [8].

Furthermore, the values of IWEP in 2019 and 2020 varied between $53 \%-55 \%$ and $55 \%-56 \%$, respectively. And the values of WSIPP in 2019 and 2020 varied between $40 \%$ $41 \%$ and $42 \%-44 \%$, respectively. None of them has 0 values, which avoids the distortion phenomenon discovered by Qian et al. [9] and Zhu et al. [10].

From the perspective of dispersion degree, the discrimination of IWEP is slightly higher than that of WSIPP in 2019 and a little lower than that of WSIPP in 2020. Therefore, the weight of IWEP should be larger than that of WSIPP in 2019 and lower than that of WSIPP in 2020.

From the perspective of dynamic changing trend, both of IWEP and WSIPP in these 3 provinces show increasing trends from 2019 to 2020 . Therefore, the agricultural water conservation conditions of the study area are improved; and the comprehensive agricultural conservation indices in 2020 should be better than those in 2019 .

3.2. Results of Weights. According to the EWM, the weights are calculated and shown in Table 3.

As shown in Table 3, according to EWM, the weight of IWEP in 2019 is higher than that of WSIPP while the weight of IWEP in 2020 is less than that of WSIPP. Obviously, the distribution of weights is consistent with the discrimination degree of indicators. Furthermore, there is no distortion phenomenon in standardized values or grades recognition.
Therefore, EWM can reflect the change of the information amount with time, and the interference of subjective factors is eliminated to ensure the objectivity of the weights. Thus, in this dynamic evaluation of agricultural water conversion, the weighting results of EWM are reasonable.

\subsection{The Abnormal Phenomena in Dynamic Evaluation} Results. Based on the dynamic evaluation model discussed in Section 2.1, the agricultural water conversion condition is assessed and illustrated in Table 4.

As illustrated in Table 4, all the comprehensive watersaving indices of the 3 provinces in 2019 are higher than 50, which belong to the "Good" grade. However, all the comprehensive water-saving indices of the 3 provinces in 2020 are less than 50, which belong to the "Medium" grade. Compared with Tables 2 and 4 , it is easy to find the following abnormal phenomena. Compared with 2019, all the indicators in 2020 show improvement trends, but the comprehensive evaluation results show a deteriorating trend.

Compared with Table 3, it can be found that this abnormal phenomenon is induced by the weights of EWM. In 2019, IWEP has a higher distinction and weight than WSIPP and plays a greater role in the comprehensive evaluation. Since the measured values of IWEP are all in the "Good" level, the comprehensive evaluation results are also inclined to "Good" grade. In 2020, the differentiation and weight of IWEP are smaller than those of WSIPP, so it plays a smaller role in the overall evaluation. As a result, the comprehensive evaluation results are also inclined to "Medium" grade, which is determined by WSIPP.

Obviously, although this result is understandable from the perspective of pure mathematics, it creates confusions in agricultural engineering. The agricultural sector has invested considerably in upgrading all agricultural facilities, and all indicators are showing an improving trend; however, in the assessment, it shows a regressive trend in agricultural water saving. This not only restrains the further improvements in agricultural water conservation but also damages the credibility of managers.

\subsection{A Judgment Method for the Abnormal Phenomenon of} EWM. According to the discussion in Section 3.3, EWM in dynamic evaluation may lead to the inconsistency between the trends of the comprehensive evaluation results and the values of the indicators. In particular, this anomaly is quite different from the limitations of EWM proposed by Yan et al. [8], Qian et al. [9], and Zhu et al. [10]. It is neither due to excessive level differences nor due to distortion in the standardization process. Instead, the weight result of EWM is a reasonable reflection of the dynamic changes in the degree of data dispersion. Therefore, the measure proposed by Yan et al. [8], Qian et al. [9], and Zhu et al. [10] to test the rationality of weights according to the distribution characteristics of indicators is not applicable.

As discussed in Section 3.3, this problem is induced by the following condition. Although all the indicators are improved, the discrimination of the worse indicators becomes larger while the discrimination of the better 
TABle 2: The data of each indicator.

\begin{tabular}{lcccccc}
\hline \multirow{2}{*}{ Measured data } & \multicolumn{3}{c}{2019} & & \multicolumn{2}{c}{2020} \\
& Province 1 (\%) & Province 2 (\%) & Province 3 (\%) & Province 1 (\%) & Province 2 (\%) & Province 3 (\%) \\
\hline IWEP & 53 & 53 & 55 & 55 & 56 & 56 \\
WSIPP & 40 & 41 & 41 & 42 & 42 & 44 \\
\hline
\end{tabular}

TABLE 3: Weighting process of EWM.

\begin{tabular}{|c|c|c|c|c|c|}
\hline \multirow{2}{*}{ Evaluating indicators } & \multicolumn{3}{|c|}{ Standardized values } & \multirow{2}{*}{ Entropy results } & \multirow{2}{*}{ Weight parameters } \\
\hline & Province 1 & Province 2 & Province 3 & & \\
\hline \multicolumn{6}{|l|}{2019} \\
\hline IWEP & 0.00 & 0.00 & 1.00 & 0.00 & 0.73 \\
\hline WSIPP & 0.00 & 1.00 & 1.00 & 0.63 & 0.27 \\
\hline \multicolumn{6}{|l|}{2020} \\
\hline IWEP & 0.00 & 1.00 & 1.00 & 0.63 & 0.27 \\
\hline WSIPP & 0.00 & 0.00 & 1.00 & 0.00 & 0.73 \\
\hline
\end{tabular}

TABLE 4: Agricultural water conversion evaluation results.

\begin{tabular}{|c|c|c|c|c|c|c|c|}
\hline \multirow{2}{*}{\multicolumn{2}{|c|}{ Evaluation projects }} & \multicolumn{3}{|c|}{2019} & \multicolumn{3}{|c|}{2020} \\
\hline & & Province 1 & Province 2 & Province 3 & Province 1 & Province 2 & Province 3 \\
\hline \multirow{2}{*}{ Water-saving index } & IWEP & 57.5 & 57.5 & 62.5 & 62.5 & 65.0 & 65.0 \\
\hline & WSIPP & 37.5 & 38.75 & 38.75 & 40.0 & 40.0 & 42.5 \\
\hline \multirow{2}{*}{\multicolumn{2}{|c|}{$\begin{array}{l}\text { Comprehensive water } \\
\text { conservation index } \\
\text { Comprehensive grade }\end{array}$}} & 52.11 & 52.45 & 56.1 & 46.07 & 46.74 & 48.57 \\
\hline & & Good & Good & Good & Medium & Medium & Medium \\
\hline
\end{tabular}

indicators becomes smaller. Therefore, we propose the following judgment method for the abnormal phenomenon of EWM.

(i) Make the trend analysis of the observation data and focus on whether all the indicators have improvement trends.

For example, as illustrated in Table 2, the IWEP of these 3 provinces is improved from $53 \%, 53 \%$, and $55 \%$ to $55 \%, 56 \%$, and $56 \%$, respectively. And their WSIPP is improved from $40 \%, 41 \%$, and $41 \%$ to $42 \%$, $42 \%$, and $42 \%$, respectively. Logically speaking, the comprehensive evaluation results should show an improvement trend, too.

(ii) Make the trend analysis of the entropy values. When the entropies of the better indicators are increasing and the entropies of the worse indicators are decreasing, EWM should not be used for assigning weights.

For example, as illustrated in Table 2, IWEP is better than WSIPP. However, as shown in Table 3, the entropies of IWEP increase from 0.00 to 0.63 while the entropies of WSIPP decrease from 0.63 to 0.00 . This situation will make the weights of worse indicators become larger while the weights of the better indicators become smaller, which finally causes the abnormal phenomenon in the dynamic evaluation results. As listed in Table 3, the weights of IWEP decrease from 0.73 to 0.27 while the weights of WSIPP increase from 0.27 to 0.73 . And as a result, the comprehensive water conservation indices of all the provinces show a deteriorating trend.

\section{Conclusion}

In this agricultural water conservation evaluation, both of IWEP and WSIPP have improvement trends in all the provinces. The IWEP of these 3 provinces is improved from $53 \%, 53 \%$, and $55 \%$ to $55 \%, 56 \%$, and $56 \%$, respectively. And their WSIPP is improved from $40 \%, 41 \%$, and $41 \%$ to $42 \%$, $42 \%$, and $42 \%$, respectively. However, according to EWM, the weights of IWEP decrease from 0.73 to 0.27 while the weights of WSIPP increase from 0.27 to 0.73 . As a result, their comprehensive agricultural conservation indices deteriorate from 52.11, 52.45, and 56.1 to 46.07, 46.74, and 48.57 , respectively. These abnormal phenomena of EWM are induced by that though all the indicators are improved, the discrimination of the worse indicators becomes larger while the discrimination of the better indicators becomes smaller.

The abnormal phenomena of EWM in dynamic evaluation can be avoided by the trend analysis of the observation data and entropy values. When all the indicators have improvement trends, but the entropies of the better indicators are increasing and the entropies of the worse indicators are decreasing, EWM should not be used for assigning weights. 


\section{Data Availability}

The data used to support the findings of this study are included within the article.

\section{Conflicts of Interest}

The authors declare that they have no conflicts of interest.

\section{Acknowledgments}

This work was supported by the Water Conservancy Fund Project of Hunan Province (XSKJ2019081-32), the National Natural Science Foundation of China (52069012) and 2021 Annual Scientific Research Program of Wanjiazhai Water Holding Group Co., Ltd. (2021-31).

\section{References}

[1] S. Z. Huang, B. Ming, Q. Huang, G. Y. Leng, and B. B. Hou, “A case study on a combination NDVI forecasting model based on the entropy weight method," Water Resources Management, vol. 31, no. 11, 2017.

[2] M. M. Sahoo, K. C. Patra, J. B. Swain, and K. K. Khatua, "Evaluation of water quality with application of bayes' rule and entropy weight method," European Journal of Environmental and Civil Engineering, vol. 21, no. 6, pp. 730-752, 2017.

[3] R. F. Dyer and E. H. Forman, "Group decision support with the analytic hierarchy process," Decision Support Systems, vol. 8, no. 2, pp. 99-124, 1992.

[4] H. N. Qin, D. R. Luo, and K. Guney, "New uncertainty measure of rough fuzzy sets and entropy weight method for fuzzy-target decision-making Tables," Journal of Applied Mathematics, vol. 2014, Article ID 487036, 7 pages, 2014.

[5] S. Q. Xu, Z. G. Hu, Q. Liu, H. Huang, and J. P. Pu, "Multiobjective decision analysis of diversion standards based on entropy," China Rural Water and Hydropower, vol. 28, no. 8, pp. 45-47, 2004.

[6] Z. H. Zou, J. N. Sun, and G. P. Ren, "Study and application on the entropy method for determination of weight of evaluating indicators in fuzzy synthetic evaluation for water quality assessment," Acta Scientiae Circumstantiae, vol. 25, no. 4, pp. 552-556, 2005.

[7] X. Q. Zhang and C. Liang, "Application of fuzzy matter-element model based on coefficients of entropy in comprehensive evaluation of water quality," Journal of Hydraulic Engineering, vol. 36, no. 9, pp. 1057-1061, 2005.

[8] F. Yan, F. H. Yi, and L. Chen, "Improved entropy weighting model in water quality evaluation," Water Resources Management, vol. 33, no. 6, 2019.

[9] B. Qian, Y. X. Zhu, Y. X. Wang, and F. Yan, "Can entropy weight method correctly reflect the distinction of water quality indices?" Water Resources Management, vol. 34, no. 573, 2020.

[10] Y. X. Zhu, D. Z. Tian, and F. Yan, "Effectiveness of entropy weight method in decision-making," Mathematical Problems in Engineering, vol. 20205 pages, Article ID 3564835, 2020.

[11] Y.-Jj. Guo, Y. Yao, and P.-T Yi, "Method and application of dynamic comprehensive evaluation," Systems EngineeringTheory \& Practice, vol. 27, no. 10, pp. 154-158, 2007.

[12] W. Y. Qian and Y. Dong, "Dynamic multi-attribute decisionmaking model and application with interval number based on improved vector similarity," Control and Decision, vol. 34, no. 01, pp. 25-30, 2019.
[13] China Renewable Energy Engineering Institute, The Chinese Water Conservation Evaluation Report, China Renewable Energy Engineering Institute, Beijing ,China, 2020. 